\title{
Comparative transcriptome analysis of the newly discovered insect vector of the pine wood nematode in China, revealing putative genes related to host plant adaptation
}

\author{
Zehai Hou' ${ }^{1}$, Fengming Shi ${ }^{1}$, Sixun Ge${ }^{1}$, Jing Tao ${ }^{1}$, Lili Ren ${ }^{1}$, Hao Wu ${ }^{2}$ and Shixiang Zong ${ }^{1 *}$
}

\begin{abstract}
Background: In many insect species, the larvae/nymphs are unable to disperse far from the oviposition site selected by adults. The Sakhalin pine sawyer Monochamus saltuarius (Gebler) is the newly discovered insect vector of the pine wood nematode (Bursaphelenchus xylophilus) in China. Adult M. saltuarius prefers to oviposit on the host plant Pinus koraiensis, rather than P. tabuliformis. However, the genetic basis of adaptation of the larvae of $M$. saltuarius with weaken dispersal ability to host environments selected by the adult is not well understood.

Results: In this study, the free amino and fatty acid composition and content of the host plants of $M$. saltuarius larvae, i.e., P. koraiensis and P. tabuliformis were investigated. Compared with P. koraiensis, P. tabuliformis had a substantially higher content of various free amino acids, while the opposite trend was detected for fatty acid content. The transcriptional profiles of larval populations feeding on P. koraiensis and $P$. tabuliformis were compared using PacBio Sequel II sequencing combined with Illumina sequencing. The results showed that genes relating to digestion, fatty acid synthesis, detoxification, oxidation-reduction, and stress response, as well as nutrients and energy sensing ability, were differentially expressed, possibly reflecting adaptive changes of $M$. saltuarius in response to different host diets. Additionally, genes coding for cuticle structure were differentially expressed, indicating that cuticle may be a potential target for plant defense. Differential regulation of genes related to the antibacterial and immune response were also observed, suggesting that larvae of $M$. saltuarius may have evolved adaptations to cope with bacterial challenges in their host environments.

Conclusions: The present study provides comprehensive transcriptome resource of $M$. saltuarius relating to host plant adaptation. Results from this study help to illustrate the fundamental relationship between transcriptional plasticity and adaptation mechanisms of insect herbivores to host plants.
\end{abstract}

Keywords: Cerambycidae, Monochamus saltuarius, Host adaptation, Transcriptional variation, Pinus koraiensis, Pinus tabuliformis

\footnotetext{
* Correspondence: zongshixiang@bjfu.edu.cn

${ }^{1}$ Key Laboratory of Beijing for the Control of Forest Pests, Beijing Forestry University, Beijing, China

Full list of author information is available at the end of the article
}

(c) The Author(s). 2021 Open Access This article is licensed under a Creative Commons Attribution 4.0 International License, which permits use, sharing, adaptation, distribution and reproduction in any medium or format, as long as you give appropriate credit to the original author(s) and the source, provide a link to the Creative Commons licence, and indicate if changes were made. The images or other third party material in this article are included in the article's Creative Commons licence, unless indicated otherwise in a credit line to the material. If material is not included in the article's Creative Commons licence and your intended use is not permitted by statutory regulation or exceeds the permitted use, you will need to obtain permission directly from the copyright holder. To view a copy of this licence, visit http://creativecommons.org/licenses/by/4.0/ The Creative Commons Public Domain Dedication waiver (http://creativecommons.org/publicdomain/zero/1.0/) applies to the data made available in this article, unless otherwise stated in a credit line to the data. 


\section{Background}

For insect herbivores, adaptation to host plants is crucial to their ability to colonize a variety of environments [1]. Host plants produce a variety of allelochemicals including various defense compounds that protect them against herbivores; meanwhile, insect herbivores have developed different means to struggle with the chemical barriers that deter them from feeding [2]. Owing to the variety of plant defense compounds, a generalist herbivorous insect has to overcome a range of chemical challenges [3]. The capacity of herbivores to metabolize and detoxify plant chemicals is considered as one of their main evolutionary adaptations [4]. Although the importance of insect adaptation to plant chemicals is widely recognized, the underlying genetic mechanisms in response to their host plant defenses are still insufficient $[3,4]$.

The pine wood nematode (PWN; Bursaphelenchus xylophilus) is a plant parasitic nematode and major cause of pine wilt disease in Asia and Europe [5]. The transfer of PWN between host trees is mediated by insect vectors, e.g., various species of Monochamus beetles $[6,7]$. In Asia, PWN infection mainly occurs during feeding and oviposition of the Japanese pine sawyer Monochamus alternatus Hope [5]. Besides M. alternatus, the Sakhalin pine sawyer M. saltuarius (Gebler) (Coleoptera: Cerambycidae) is another important insect vector of PWN in Japan [8] and Korea [9]. Recently, M. saltuarius has also been confirmed as an effective vector of PWN in Liaoning Province, China [10-12]. The Korean white pine Pinus koraiensis Siebold \& Zucc, a tree species of economic importance [13], was found to be a natural host for the PWN in the Republic of Korea in 2006, and M. saltuarius transmitted PWN to P. koraiensis [14]. Similarly, M. saltuarius was found to transmit PWN to P. koraiensis in China $[10,12]$. In addition, Han et al. [15] investigated the feeding and oviposition preference of $M$. saltuarius among eight tree species, including $P$. koraiensis, and they found that the highest feeding amount and oviposition preference were related to $P$. koraiensis. Similarly, Pan et al. [16] reported that adults of $M$. saltuarius preferred P. koraiensis than P. tabuliformis Carr. and Larix kaempferi (Lamb.) Carr. based on feeding behavior. Volatiles produced by host plants, e.g., $\alpha$-pinene, are known to attract Monochamus spp. [17, 18]. Adults of $M$. saltuarius can be attracted by terpenes emitted from the host plant $P$. koraiensis for feeding and oviposition $[19,20]$. In addition, host volatiles also play an important role in the mating location of longhorned beetles [21]. Therefore, the distribution pattern in the adults of $M$. saltuarius can be affected by host volatiles.

In many organisms, including insect species, larvae/ nymphs are unable to disperse far from the oviposition site selected by the mother [22]. Consequently, oviposition host selection can strongly impact both the survival and the spatial distribution of a species [23], and the structure and composition of animal communities [24]. Female adults of $M$. saltuarius lay their eggs on the bark of pine trees. After hatching, the larvae feed on the inner cambium bark and outer sapwood. Because adults of $M$. saltuarius prefer $P$. koraiensis over $P$. tabuliformis for feeding and oviposition, coupled with the weakened dispersal ability at the instar stage, the larvae of $M$. saltuarius may be confronted with different chemical challenges posed by their different hosts. However, the molecular mechanisms underlying host plant adaptation of $M$. saltuarius larvae are largely unknown.

Detecting transcriptional changes related to host adaptation is a vital link to understand plant-insect interactions [3, 25, 26]. Previous studies have proved that transcriptional plasticity of insects was related to diet. For instance, research on host adaptation in cactophilic flies, e.g., Drosophila mojavensis, D. buzzatii, and D. mettleri, have identified a series of genes associated with carbohydrate metabolism, cellular energy production, xenobiotic metabolism, and stress response [2, 25, 27]. Research on the striped stem borer Chilo suppressalis, Zhong et al. [26] identified several genes involved in host plant adaptation processes, including digestion and detoxification. Larvae of the Asian long-horned beetle Anoplophora glabripennis modulate a subset of genes associated with digestion when fed on a nutrient-poor, compared to a nutritious diet [28]. In addition, Scully et al. [29] showed that feeding on two appropriate host plants (Acer spp. and Populus nigra) modified the expression levels of multicopy genes involved in digestion and detoxification in A. glabripennis. Recently, Hou \& Wei [30] examined the transcriptional changes of the cicada Subpsaltria yangi, on a varied diet of different host plants. The authors suggested that gene expression changes, relating to digestion, detoxification, oxidoreductase metabolism, and stress response, may be a vital adaptation to diet and habitat.

With the rapid development of sequencing technology, research into the insect transcriptome is increasing [31, 32]. However, de novo transcriptome assembly represents a challenge for non-model insect species, because it generally relies on the use of short cDNA sequences (such as Illumina technology). Recently, single-molecule real-time sequencing (SMRT-seq) technology has been applied to generate long sequence reads, allowing the production of full-length transcripts without assembly algorithms [33]. SMRT-seq has been reported to provide inaccurate information on genes, which could be calibrated based on Illumina reads from matched samples [34]. Therefore, the combination of SMRT-seq and Illumina RNA-seq can be used to obtain comprehensive genetic information, including for the detection of gene isoforms and functional variants $[35,36]$. 
In the present study, the free amino and fatty acid composition and content of the two host plants of $M$. saltuarius, categorized as either the "preferred" P. koraiensis or "non-preferred" P. tabuliformis, was investigated. The genome-wide transcriptional profiles of $M$. saltuarius larvae feeding on $P$. koraiensis and P. tabuliformis was compared by combining SMRT-seq and Illumina RNA-seq analysis. Our aim was to identify differentially expressed genes (DEGs) in $M$. saltuarius relating to host plant adaptation based on diet. The results provide new information for further research on the mechanisms underlying transcriptional plasticity and adaptation of insect herbivores to different host plants. Furthermore, understanding the molecular differences of $M$. saltuarius when feeding on different hosts may provide significant enlightenment for the arrangement of host resistance in the control of PWN transmission.

\section{Results}

\section{Host plant free amino and fatty acid composition and} content

Eight free amino acids were found in P. koraiensis, including glutamic acid (Glu), aspartic acid (Asp), threonine (Thr), lysine (Lys), alanine (Ala), serine (Ser), valine (Val), and glycine (Gly). Twelve free amino acids were found in P. tabuliformis, i.e., Glu, Asp, leucine (Leu), Thr, Lys, Ala, Ser, Val, proline (Pro), Gly, isoleucine (Ile), and histidine (His). The main free amino acids in the two host plants were Glu and Asp. Compared with $P$. koraiensis, $P$. tabuliformis had a substantially higher content of most free amino acids (Fig. 1a).

Twenty-nine and thirty fatty acids were detected in $P$. koraiensis and P. tabuliformis, respectively. The predominant fatty acids present in the two host plants were linoleic $(\mathrm{C} 18: 2 \mathrm{n} 6 \mathrm{c})$, oleic $(\mathrm{C} 18: 1 \mathrm{n} 9 \mathrm{c})$, and palmitic acids
(C16:0). Compared with P. tabuliformis, P. koraiensis had a substantially higher content of most fatty acids (Fig. 1b, c).

\section{Combined sequencing of Monochamus saltuarius transcripts}

The full-length transcriptome of $M$. saltuarius was produced based on the pooled RNA from the six samples of $M$. saltuarius using the PacBio Sequel II platform. A total of $22.36 \mathrm{~Gb}$ subreads was produced by one SMRT cell from the PacBio library (Table 1). The subreads from the same polymerase read sequence formed a circular consensus sequence (CCS), which yielded 284,546 CCSs with an average read length of $2583 \mathrm{bp}$, and the length distribution of the CCS reads is shown in Additional file 1: Figure S1a. Among them, 234,939 full-length non-chimera (FLNC) reads $(82.57 \%$ of CCSs) were obtained, and the length distribution of the FLNC reads is shown in Additional file 1: Figure S1b. In total, 48,361 consensus isoforms with a mean length of $3122 \mathrm{bp}$ were detected through the Iterative Clustering for Error Correction (ICE), including 46,082 polished highquality isoforms (Table 1). The 48,361 consensus isoforms were corrected based on the Illumina RNA-seq data (Table 2) to improve quality. After removing redundant sequences and a cluster of low-quality transcripts using CD-HIT (c=0.99), a total of 32,304 non-redundant transcripts with a mean length of $3290 \mathrm{bp}$ were obtained, which were further annotated for downstream analysis. The completeness of our transcript dataset was assessed with benchmarking universal single-copy orthologs (BUSCO), and the result revealed that this dataset consisted of $89.5 \%$
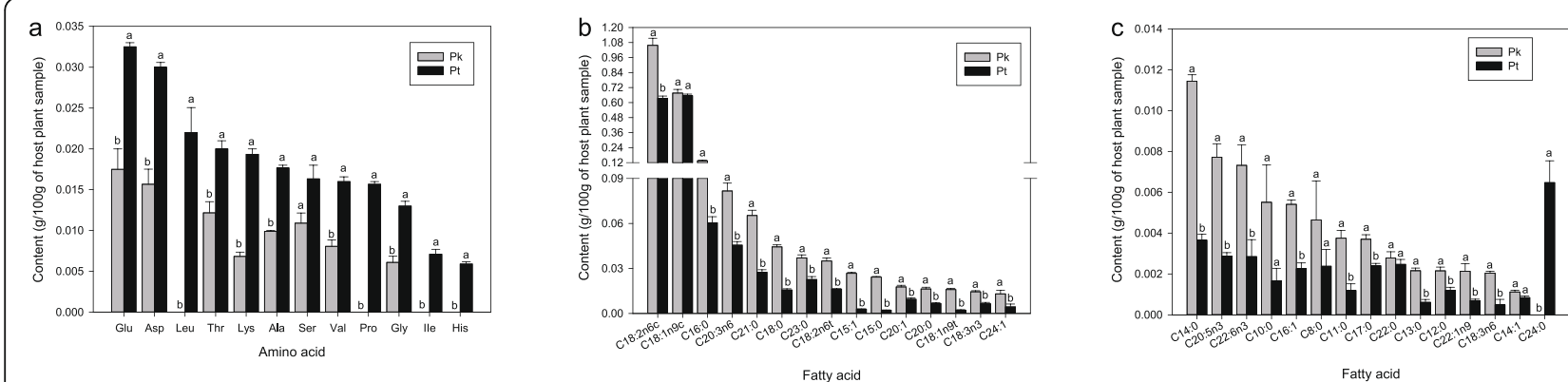

Fig. 1 Amino acid and fatty acid composition and content between host plants Pinus koraiensis and P. tabuliformis. a Amino acid. b, c Fatty acid. Glu, glutamic acid; Asp, aspartic acid; Leu, leucine; Thr, threonine; Lys, lysine; Ala, alanine; Ser, serine; Val, valine; Pro, proline; Gly, glycine; lle, isoleucine; His, histidine. C18:2n6c, linoleic acid; C18:1n9c, oleic acid; C16:0, palmitic acid; C20:3n6, Dihomo-y-linolenic acid; C21:0, Heneicosylic acid; C18:0, Stearic acid; C23:0, Tricosanoic acid; C18:2n6t, Linoelaidic acid; C15:1, 10c-pentadecenoic acid; C15:0, Pentadecanoic acid; C20:1, Eicosenoic acid; C20:0; Arachidic acid; C18:1n9t, Elaidic acid; C18:3n3, a-Linolenic acid; C24:1, Nervonic acid; C14:0, Myristic acid; C20:5n3, Eicosapentaenoic acid (EPA); C22:6n3, Docosahexaenoic acid (DHA); C10:0, Decanoic acid; C16:1, Palmitoleic acid; C8:0, Octanoic acid; C11:0, Undecanoic acid; C17:0, Margaric acid; C22:0, Behenic acid; C13:0, Tridecylic acid; C12:0, Lauric acid; C22:1n9, Erucic acid; C18:3n6, Y-Linolenic acid; C14:1, Myristoleic acid; C24:0, Lignoceric acid. Data are shown as mean \pm SE. Different letters represent significant statistical difference at the 0.05-level 
Table 1 Summary for the full-length transcriptome of Monochamus saltuarius analyzed with the PacBio Sequel II platform

\begin{tabular}{ll}
\hline Library & $\mathbf{1 - 6} \mathbf{k b}$ \\
\hline SMRT cell & 1 \\
Subreads base (G) & 22.36 \\
Number of CCS & 284,546 \\
Read bases of CCS & $735,095,084$ \\
Mean read length of CCS & 2583 \\
Mean number of passes & 36 \\
Number of undesired primer reads & 37,473 \\
Number of filtered short reads & 21 \\
Number of full-length non-chimeric reads & 234,939 \\
Number of consensus isoforms & 48,361 \\
Average consensus isoforms read length (bp) & 3122 \\
Number of polished high-quality isoforms & 46,082 \\
Number of polished low-quality isoforms & 1917 \\
Number of non-redundant transcripts & 32,304 \\
\hline
\end{tabular}

complete and $1.9 \%$ partial BUSCO orthologs (Additional file 2: Figure S2).

For Illumina sequencing, $36.91 \mathrm{~Gb}$ high quality sequences were obtained from the six mRNA samples of $M$. saltuarius. The guanine-cytosine (GC) content of data sequenced from the six libraries was $\sim 42 \%$, and the percentage of reads with an average quality score $>30$ was above 93\% (Table 2). This result indicated that the accuracy and quality of the sequenced data were sufficient for further analysis. The Illumina sequencing reads were not assembled alone because more than $85 \%$ of them mapped to the 32,304 non-redundant transcripts (Table 2).

\section{Functional annotation}

To obtain a comprehensive functional annotation of the full-length transcriptome of $M$. saltuarius, a total of 32, 304 non-redundant transcripts were aligned with different databases (Table 3). A total of 29,798 transcripts (92.24\%) were annotated in at least one database. The transcripts were mostly annotated by the $\mathrm{Nr}$ (NCBI non-redundant protein sequences) database $(29,113 ; 90.12 \%)$ (Additional file 3: Table S1). The highest percentage of unigene sequences were matched with Anoplophora glabripennis (83.18\%), followed by Leptinotarsa decemlineata (2.04\%), Tribolium castaneum (1.80\%), and Callosobruchus maculatus (1.51\%) (Additional file 4: Figure S3).

In total, 13,144 transcripts were assigned Gene Ontology (GO) terms, which were classified into the three major GO categories (Additional file 5: Figure S4). For the biological process classification, genes involved in 'cellular process', 'single-organism process', and 'metabolic process' were highly represented. For the cellular component, the major categories were 'cell', 'cell part', and 'organelle'. For the molecular function classification, 'binding' was the most enriched GO term, followed by 'catalytic activity'. Kyoto Encyclopedia of Genes and Genomes (KEGG) analysis shows that the matched 27,077 transcripts are assigned into 336 pathways. The most wellrepresented metabolic pathways are involved in 'global and overview maps', 'carbohydrate metabolism', 'lipid metabolism', and 'amino acid metabolism' (Additional file 6: Figure S5).

Table 2 Illumina-sequencing data analysis results

\begin{tabular}{|c|c|c|c|c|c|c|c|}
\hline ID & $\begin{array}{l}\text { Read } \\
\text { number }\end{array}$ & $\begin{array}{l}\text { Base } \\
\text { number }\end{array}$ & $\begin{array}{l}\text { GC content } \\
(\%)\end{array}$ & $\begin{array}{l}\text { Q30 } \\
(\%)\end{array}$ & $\begin{array}{l}\text { Uniquely mapped } \\
\text { reads (\%) }\end{array}$ & $\begin{array}{l}\text { Reads mapped to multiple } \\
\text { loci }(\%)\end{array}$ & $\begin{array}{l}\text { Reads mapped to many } \\
\text { loci }(\%)\end{array}$ \\
\hline Pk1 & $19,908,903$ & $\begin{array}{l}5,943,030 \\
832\end{array}$ & 41.98 & 93.18 & 36.57 & 42.86 & 6.13 \\
\hline Pk2 & $20,814,420$ & $\begin{array}{l}6,198,988 \\
604\end{array}$ & 42.01 & 93.49 & 35.25 & 44.10 & 7.28 \\
\hline Pk3 & $19,898,375$ & $\begin{array}{l}5,936,828 \\
046\end{array}$ & 42.02 & 93.59 & 35.06 & 43.17 & 7.80 \\
\hline Pt1 & $20,331,901$ & $\begin{array}{l}6,064,964 \\
864\end{array}$ & 42.17 & 93.38 & 37.87 & 42.08 & 6.29 \\
\hline Pt2 & $21,214,464$ & $\begin{array}{l}6,338,924 \\
668\end{array}$ & 42.65 & 93.29 & 35.45 & 43.17 & 8.99 \\
\hline Pt3 & $21,507,511$ & $\begin{array}{l}6,426,164 \\
508\end{array}$ & 42.49 & 93.30 & 38.79 & 41.75 & 6.28 \\
\hline
\end{tabular}

Q30: proportion of nucleotides with quality value larger than 30 in reads. This means that the base call accuracy (i.e., the probability of a correct base call) is $99.9 \%$ 
Table 3 Non-redundant transcripts identified from different databases

\begin{tabular}{ll}
\hline Annotated databases & Number \\
\hline $\mathrm{Nr}$ & 29,113 \\
KEGG & 27,077 \\
eggNOG & 26,783 \\
$\mathrm{NT}$ & 25,234 \\
Pfam & 22,435 \\
KOG & 20,446 \\
Swiss-Prot & 20,412 \\
GO & 13,144 \\
At least one database & 29,798 \\
All database & 9262 \\
\hline
\end{tabular}

\section{Transcription factor identification, and IncRNA and SSR prediction}

A total of 1833 transcription factors (TFs) were identified, with zf-C2H2 accounting for the largest proportion of the known TF families, followed by ZBTB (Additional file 7: Figure S6). Four coding potential analysis methods were used to predict the long non-coding RNA (lncRNA), including coding potential calculator (CPC), coding-noncoding index $(\mathrm{CNCI})$, coding potential assessment tool (CPAT), and protein family (Pfam) database. The numbers of IncRNAs predicted from non-redundant transcripts by CPC, CNCI, CPAT, and Pfam were 5841, 11,740, 8203 and 8899 , respectively (Fig. 2). The intersection of these four results yielded 4455 lncRNA transcripts (Fig. 2). The average length of the lncRNA transcripts was $2863 \mathrm{bp}$.

In this study, 31,530 transcripts were scanned by MISA (MIcroSAtellite identification tool). A total of 17, 164 simple sequence repeats (SSRs) were identified from 10,929 transcripts, including six major subtypes: mononucleotide $(12,875)$, di-nucleotide (1838), tri-nucleotide (2231), tetranucleotide (187), penta-nucleotide (21), and hexa-nucleotide (12). Among them, 1398 SSRs were present in the compound formation (Additional file 8: Table S2).

\section{DEG analysis}

We evaluated the differences in gene expression between the population feeding on P. koraiensis and P. tabuliformis. It resulted in 2166 DEGs identified in the larvae of $M$. saltuarius feeding on $P$. tabuliformis $(\mathrm{Pt})$ compared with $P$. koraiensis (Pk), including 970 upregulated genes and 1196 downregulated genes (Additional file 9: Table S3; Additional file 10: Figure S7).

In this study, transcriptional changes related to host plant adaptation in $M$. saltuarius was the main focus. We identified 21 DEGs associated with digestion in the comparative set 'Pt vs Pk', encoding three carbohydrases and 18 proteases. Most of these were upregulated in $P$. tabuliformis (Fig. 3a). In addition, we identified 12 DEGs related to protease inhibitor, including eight serine proteases and four trypsin inhibitors (Fig. 3a).

Solute carriers (SLC) are a group of membrane transport proteins, which mediate the transport of various

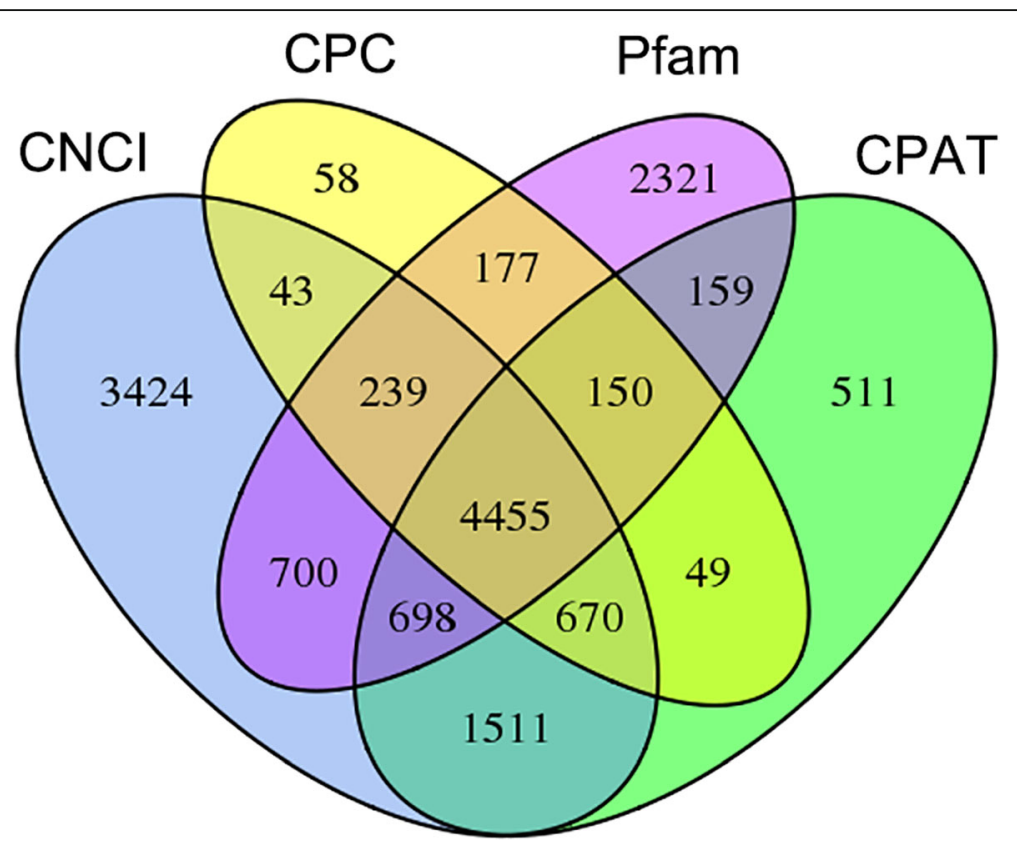

Fig. 2 Venn diagram of the number of IncRNAs predicted using coding-non-coding index (CNCI), coding potential calculator (CPC), coding potential assessment tool (CPAT) and protein family (Pfam) database 


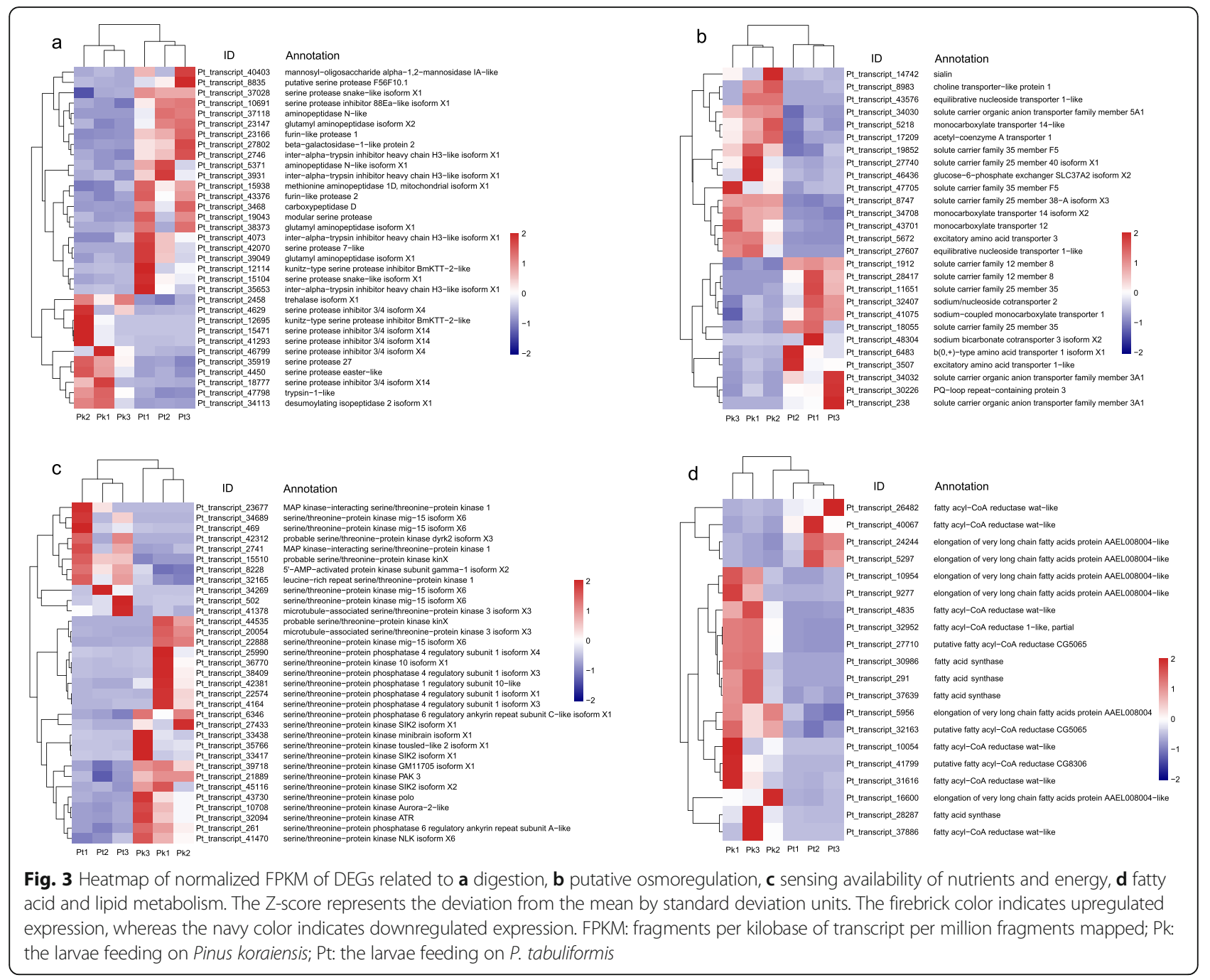

substrates across cells, including ions, nucleotides, sugars, and amino acids. We identified 27 DEGs encoding solute carriers in the comparative set ' $\mathrm{Pt}$ vs $\mathrm{Pk}$ ' (Fig. 3b), which may mediate the influx or efflux of substance and involve in the osmoregulation in the host adaptation of $M$. saltuarius.

The serine/threonine protein kinase (STK) target of rapamycin, a central element of an evolutionarily conserved eukaryotic signaling pathway, is known to act as a central regulator of cell metabolism and to respond to growth factors and nutritional status. In the present study, we identified 25 DEGs encoding STKs and seven DEGs encoding serine/threonine phosphatases (STPs) (Fig. 3c). In addition, AMP-activated protein kinase (AMPK) serves as an important regulator of cellular metabolism and energy balance. One gene encoding AMPK was found upregulated in the comparative set 'Pt vs Pk' (Fig. 3c).

Fatty acids are a significant energy store for insects. Four DEGs encoding fatty acid synthase (FAS) were identified in the comparative set 'Pt vs Pk' (Fig. 3d). In addition, six genes encoding elongation of very long chain fatty acids protein (ELOVL) were differentially expressed in the population feeding on $P$. tabuliformis when compared with P. koraiensis (Fig. 3d). Besides FAS and ELOVL, fatty acyl-CoA reductase (FAR), which can convert fatty acids to alcohols, performs a crucial role in lipid synthesis and metabolism. Ten DEGs encoding FARs were identified in the comparative set ' $\mathrm{Pt}$ vs $\mathrm{Pk}$ ', including eight upregulated in the population feeding on P. tabuliformis (Fig. 3d).

Insect herbivores should be able to deal with defense compounds and adverse environment when obtaining nutrients from their host plants. In the present study, detoxification-related DEGs were identified, including 11 cytochrome P450 monooxygenases (P450s), three UDPglycosyltransferases (UGTs), seven carboxylesterases (CEs), and 14 ATP-binding cassette (ABC) transporters (Fig. 4a). Among which, ten P450s, two UGTs, six CEs, and eight $\mathrm{ABC}$ transporters were upregulated in the population feeding on $P$. tabuliformis compared with $P$. 


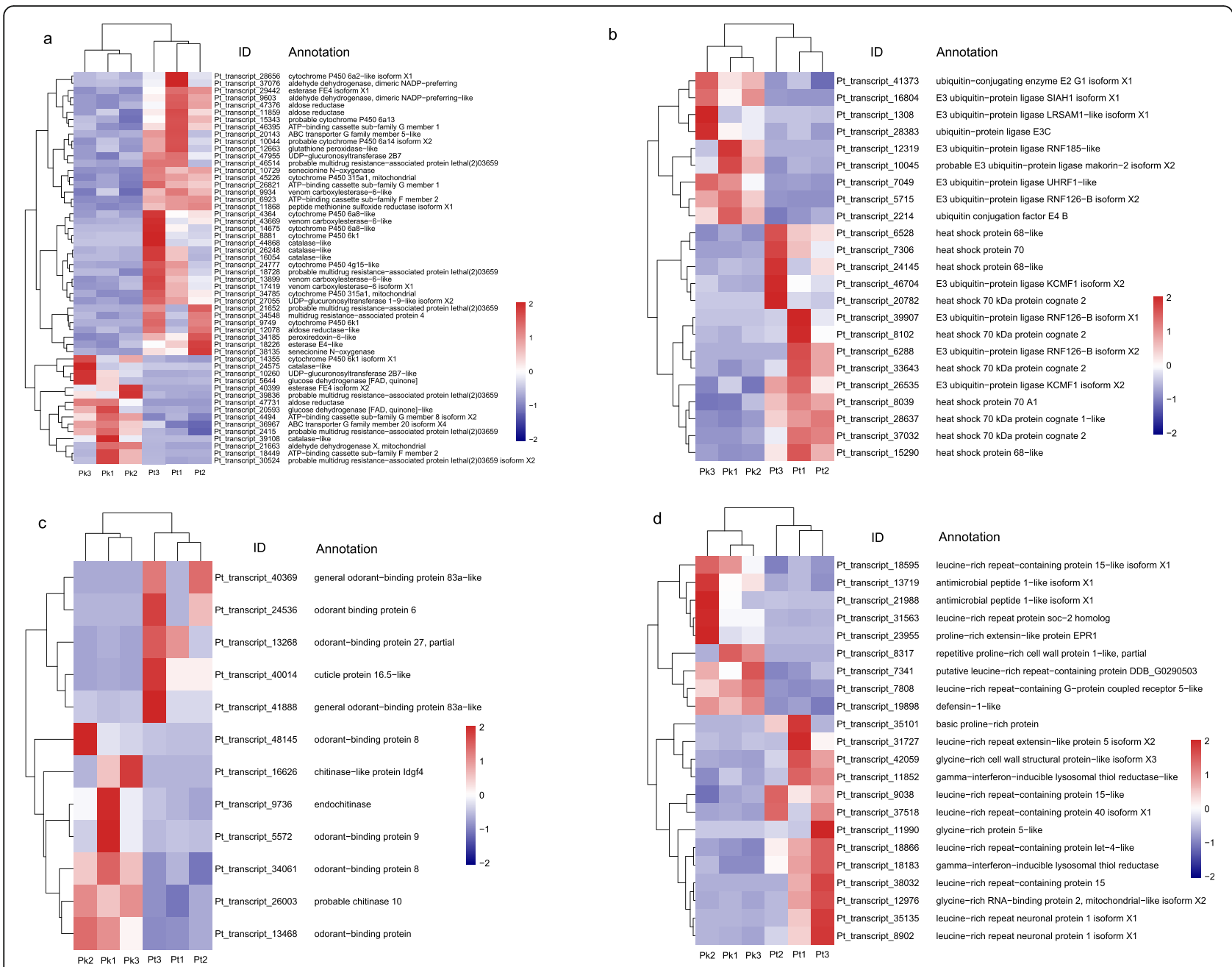

Fig. 4 Heatmap of normalized FPKM of DEGs related to a detoxification and oxidation-reduction, $\mathbf{b}$ stress response, $\mathbf{c}$ structural and general odorant binding proteins, $\mathbf{d}$ antibacterial and immune response. The Z-score represents the deviation from the mean by standard deviation units. The firebrick color indicates upregulated expression, whereas the navy color indicates downregulated expression. FPKM: fragments per kilobase of transcript per million fragments mapped; Pk: the larvae feeding on Pinus koraiensis; Pt: the larvae feeding on P. tabuliformis

koraiensis (Fig. 4a). We identified three aldehyde dehydrogenases (ALDHs), four aldose reductases, two senecionine N-oxygenases (SNOs), and two glucose dehydrogenases, most of which were upregulated in the population feeding on P. tabuliformis (Fig. 4a). We also found that DEGs encoding peroxidase, i.e., five catalases (CAT), one glutathione peroxidase (GPx)-like, and one peroxiredoxin (Prx)-6-like, were mainly upregulated in the population feeding on $P$. tabuliformis compared with P. koraiensis (Fig. 4a). These genes might involve in defense response against oxidative stress, e.g., reactive oxygen species (ROS) intake in the feeding behavior. In addition, we found that one peptide methionine sulfoxide reductase (MSRA) gene was upregulated in the population feeding on P. tabuliformis (Fig. 4a), which may help repair proteins inactivated by oxidation.
Heat shock family 20 and 70 proteins serve as chaperones for damaged proteins in wood-consuming insects. In the present study, ten genes encoding heat shock proteins (Hsp), including seven Hsp70 and three Hsp68, were upregulated in the population feeding on P. tabuliformis compared with P. koraiensis (Fig. 4b). Additionally, other DEGs involved in the stress response were also identified, including 11 genes encoding E3 ubiquitin ligase, one gene encoding ubiquitin conjugating enzyme E2G1, and one gene encoding ubiquitin conjugation factor E4B (Fig. 4b).

Plant-derived compounds may interfere with the production of chitin and cuticular protein, which compels insect herbivores to adjust the production of these structural constituents. In the present study, three genes encoding chitinase, and one gene encoding cuticular 
protein were differentially expressed in the population feeding on $P$. tabuliformis compared with $P$. koraiensis (Fig. 4c). Additionally, eight genes encoding odorantbinding protein/general odorant-binding protein (OBP/ GOBP) were differentially expressed in the comparative set 'Pt vs Pk' (Fig. 4c).

Insects always interact with a wide array of pathogens, e.g., pathogenic bacteria. Insects can prevent infection by synthesizing antibacterial proteins such as cecropin, insect defensin, large glycine-rich protein, small prolinerich protein, and lysozyme [37, 38]. In addition, leucinerich repeat (LRR)-containing proteins play important roles in pathogen-associated molecular pattern recognition to fight infection by pathogens. In the present study, we found two genes encoding antimicrobial peptide 1like isoform X1, one gene encoding defensin-1-like, three genes encoding glycine-rich proteins, three genes encoding proline-rich proteins, and two genes encoding gamma-interferon-inducible lysosomal thiol reductase (GILT), were differentially expressed in the comparative set 'Pt vs Pk' (Fig. 4d). Additionally, we identified 11 DEGs encoding LRR containing proteins in the population feeding on P. tabuliformis compared with P. koraiensis (Fig. 4d).

\section{Enrichment pathway analysis of DEGs}

To analyze functions of the DEGs, all were mapped to terms in the KEGG database. KEGG pathways with a $P$ value $<0.05$ are provided in Additional file 11: Table S4, including insect hormone biosynthesis (ko00981), tryptophan metabolism (ko00380), cysteine and methionine metabolism (ko00270), and glycine, serine, and threonine metabolism (ko00260) (Fig. 5). As mentioned above, the content of glycine and threonine in P. tabuliformis was higher than that in P. koraiensis (Fig. 1a). This suggests that the "glycine, serine and threonine metabolism" pathway may play a key role in the host plant adaptation of $M$. saltuarius.

Following this, GO term enrichment analysis was performed. The strongest changes in the top $20 \mathrm{GO}$ categories are shown in Additional file 12: Figure S8, including "oxidoreductase activity (GO:0016491)" of molecular function, and "alpha-amino acid metabolic process (GO:1901605)" of biological process.

\section{Validation of RNA-seq data by qRT-PCR}

To validate the RNA-seq results, the relative expression levels of 12 selected genes were analyzed by real-time quantitative PCR (qRT-PCR). The genes and primers used for qRT-PCR are shown in Additional file 13: Table S5. Among the 12 genes, the majority showed a consistent expression pattern between RNA-seq and qRT-PCR (Fig. 6a). The correlation analysis results for these detected DEGs are as follows: $y=0.3864 x+0.2839$, and $R^{2}=0.759$ (Fig. 6b), indicating that RNA-seq data were reliable.

\section{Discussion}

Herbivorous insects are hypothesized to express distinctive digestive enzymes to feed on host plants with different nutritional values [39, 40]. Indeed, some metabolismrelated genes are differentially expressed to adapt to the different biochemical compositions of host plant diets [2,

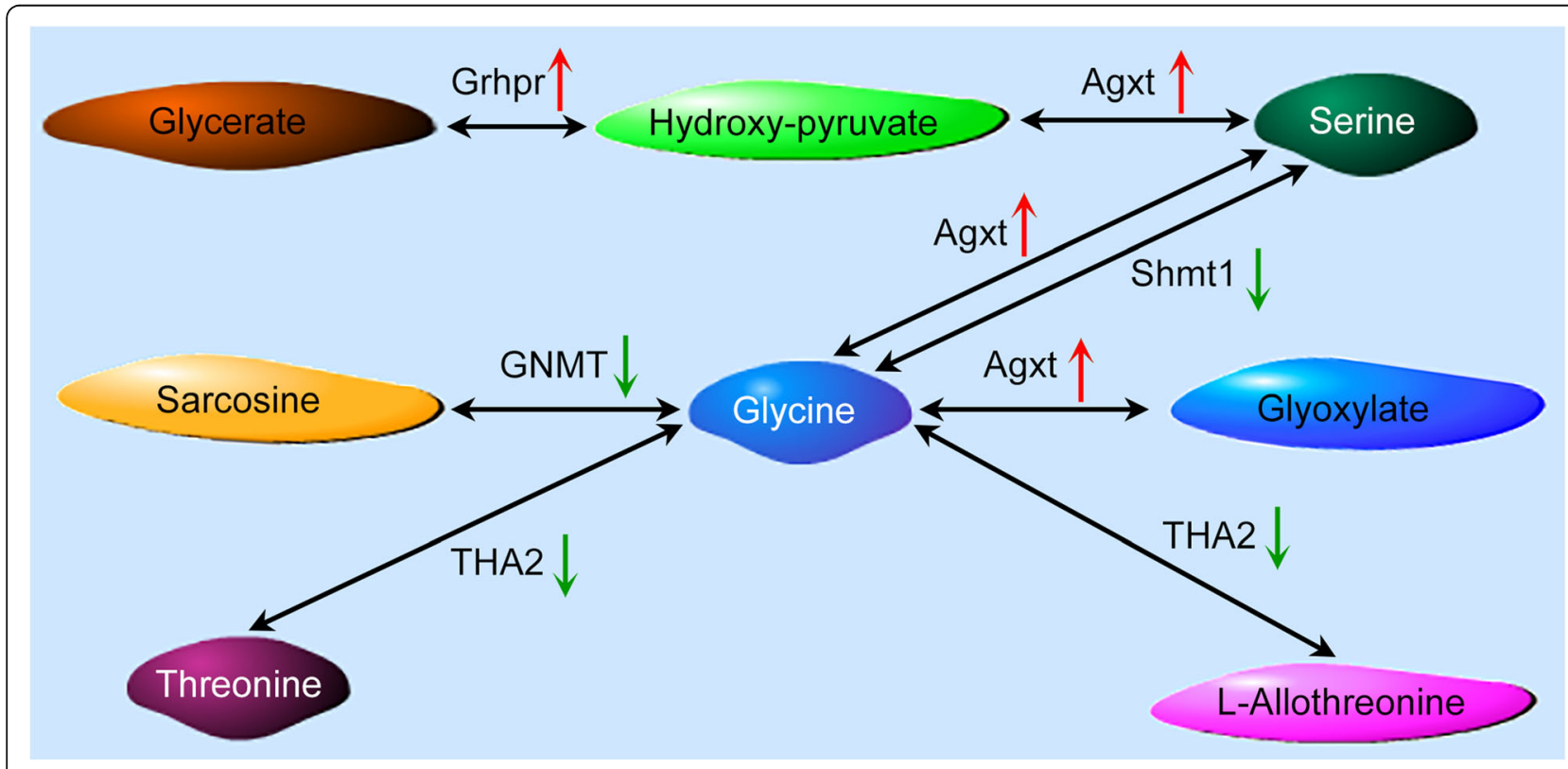

Fig. 5 DEGs involved in glycine, serine, and threonine metabolic pathways. Red and green arrows indicate significantly up- and downregulated expression, respectively 

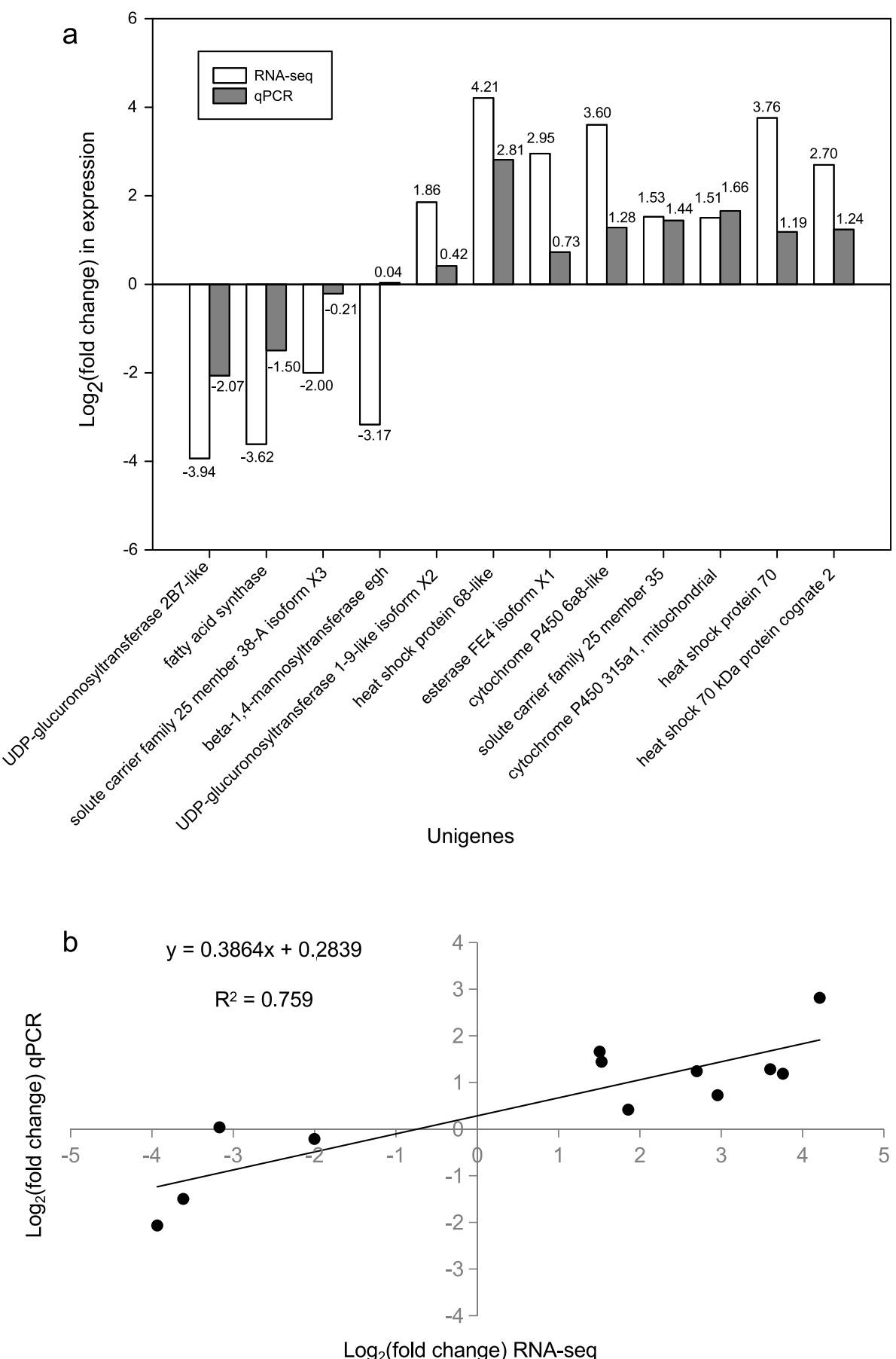

Fig. 6 Validation of sequencing data by qRT-PCR. a The expression changes for each candidate gene as measured by RNA-seq and qRT-PCR. b Scatter plot showing correlation between $\log _{2}$ (fold change) obtained via RNA-seq and qRT-PCR. Log $_{2}$ (fold change) qPCR was calculated by $-\Delta C \mathrm{Ct}=-\left[\left(C t_{p t}-\right.\right.$ $\left.\left(t_{n o m}\right)-\left(C t_{p k}-C t_{n o m}\right)\right]$

25, 41]. For instance, Chikate et al. [42] revealed dietspecific protease expression patterns in the cotton bollworm Helicoverpa armigera responding to nutritionally distinct host plants. They suggested that serine proteases play a crucial role in this polyphagous insect to adapt to a diet of many different host plants [42]. The butterfly larvae of Polygonia c-album were demonstrated to adapt similarly to host plant diet [43]. In order to examine genes related to host plant utilization, Mason et al. [28] compared gene expression changes of the larvae of Anoplophora glabripennis feeding on a preferred host (the sugar maple) to those consuming a nutrient-rich artificial diet. Recently, 
Scully et al. [29] examined how feeding on two susceptible (Acer spp. and Populus nigra) and a resistant host (Populus tomentosa) affected the gene expression of $A$. glabripennis.

In our present study, the two host plants differed in their free amino and fatty acid composition and content; thus, they were expected to have different nutritional values for $M$. saltuarius. In particular, the glycine and threonine content in the host plant $P$. koraiensis was higher than that in P. tabuliformis. KEGG pathway analysis of DEGs identified several pathways associated with amino acid metabolism, including glycine, serine and threonine metabolism. This result suggests that the different nutritional quality between the two hosts may require $M$. saltuarius to express different digestive enzymes.

The serine/threonine kinase TOR (target of rapamycin) serves as a central regulator of cell growth and cellular energy [44, 45]. Both protein kinases and their cognate phosphatases participate in sensing external stimuli [46-48]. In addition, AMPK is also known as the "energy sensor" of a cell [49]. Interestingly, AMPK and TOR are functionally interconnected, opposing signaling pathways associated with sensing the status of nutrients and energy [50]. In the present study, the genes encoding AMPK, STKs, and STPs, may be relevant to host adaptation of M. saltuarius by playing an important role in sensing the availability of nutrients and energy for the regulation of cell growth.

Fatty acids are important energy stores in insects [51] with FAS being a key enzyme for its synthesis. Animals can gain fatty acids from their diet, then generate saturated and monounsaturated fatty acids through FAScatalyzed synthesis [52-54]. Fatty acid synthesis includes cycles of four reactions, in which each cycle extends an initial acetyl-CoA by two carbons. The cycles can be repeated up to seven times to generate palmitic acid (C16: 0 ) [55]; further growth requires elongases of long or very long chain fatty acids [56]. In addition, the FAR gene family, which can convert fatty acids to fatty alcohols, also performs a crucial role in lipid synthesis and metabolism. In insects, fatty alcohols can act as precursors in the production of pheromones and cuticular hydrocarbons [57, 58]. For instance, Li et al. [59] reported that FARs are requisite for cuticle shedding, and are involved in cuticular hydrocarbon production in the destructive rice pest Nilaparvata lugens. In the present study, a substantially higher content of most fatty acids was found in the host plant $P$. koraiensis compared with $P$. tabuliformis. Moreover, four, six, and ten genes encoding FAS, ELOVL, and FAR, respectively, were differentially expressed, suggesting that they may be involved in fatty acid metabolism when M. saltuarius is confronted with different fatty acid content between the two host plants.
Besides extracting nutrients from host plants, insects need to cope with toxic chemical deterrents produced by their hosts. For instance, $P$. tabuliformis can produce defensive monoterpene, in which $\alpha$-pinene is the most abundant [60]. Besides $\alpha$-pinene, volatile organic compounds (VOCs) from $P$. tabuliformis also includes limonene, $\beta$-pinene, and $\alpha$-caryophyllene [61]. Xu et al. [62] found that the VOCs from $P$. koraiensis included $\alpha$ pinene, $\beta$-pinene, and sabinene. Insects can successfully survive on their host plants by producing detoxifying enzymes, e.g., P450s, UGTs, and CEs [25, 30, 41]. In the present study, we found that $11 \mathrm{P} 450$, seven CE, and three UGT, and most of them were upregulated in the larvae feeding on $P$. tabuliformis compared with $P$. koraiensis. Also involved in detoxification are $\mathrm{ABC}$ transporters, which act as membrane-bound proteins for the transport of various substrates across the lipid membrane, including drugs and insecticides [63, 64]. In our study, 14 genes encoding $\mathrm{ABC}$ transporters were differentially expressed, including the up-regulation of eight, in larvae feeding on $P$. tabuliformis compared with $P$. koraiensis, indicating that these genes may involve in detoxification metabolism during the host plant adaptation of $M$. saltuarius.

In our study, we found that three, two, and six genes encoding aldehyde dehydrogenases, senecionine $\mathrm{N}$-oxygenases, and aldo-keto reductases (AKRs), respectively, were differentially expressed, suggesting their involvement in plant chemical detoxification by $M$. saltuarius. The aldehyde dehydrogenases are oxidizing enzymes that are involved in detoxification of both exogenous and endogenous aldehydes [65]. In addition, senecionine $\mathrm{N}$-oxygenase, a flavindependent monooxygenase, may help insects cope with pyrrolizidine alkaloids, as observed in the larvae of the European cinnabar moth Tyria jacobaeae [66]. The AKRs are a superfamily of dehydrogenases/reductases that catalyze the synthesis and detoxification of carbonyls [67]. Previously, AKRs have been hypothesized to play an important role in the degradation of woody tissue [28]. In this study, we therefore speculate that genes encoding AKRs may not only be involved in detoxification but may also assist $M$. saltuarius to digest woody tissue.

Insect herbivores are frequently challenged with ROS which are a by-product from the metabolism of molecular oxygen [68]. Some herbivorous insects have evolved a protective response to ROS by producing detoxifying enzymes, including glutathione peroxidase (GPx), catalase (CAT), superoxide dismutase (SOD), and ascorbate peroxidase [69]. For instance, in a serious pest on wheat crops (the Hessian fly Mayetiola destructor), when feeding on resistant wheat seedlings, the larvae upregulates expression of phospholipid glutathione peroxidases, catalases, and superoxide dismutases, to counteract ROS [68]. In our study, we found that five genes encoding CATs, one 
GPx-like, and one Prx6-like gene, were upregulated in the larvae feeding on $P$. tabuliformis compared with $P$. koraiensis, suggesting that they may involve in the defense against ROS intake during feeding.

As well as battling toxic chemicals from host plants, insects also must deal with a wide array of pathogens. Insects combat infection by mounting a powerful immune response [70]. Insect immune system contains two major aspects: the cellular and humoral responses [71]. The humoral response includes synthesis of antimicrobial proteins [72], which are grouped into five main types: cecropins, insect defensins, large glycine-rich proteins, small proline-rich proteins, and lysozymes [38]. The LRR is a highly conserved motif usually consisting of 20-30 residues rich in leucine, and a LRR domain, which is an important binding component for immunerelated proteins [73]. In the insect Manduca sexta, a soluble, extracellular leucine-rich repeat protein (leureptin) could bind to bacterial lipopolysaccharide (LPS) and involve in hemocyte responses to bacterial infection [74]. GILT is involved in the bacterial immune response in various organisms [75]. Recently, empirical studies demonstrated that pinewood nematode infection increased the microbial diversity in pines [76, 77]. In the present study, genes encoding antimicrobial peptide 1-like isoform X1, defensin-1-like, glycine-rich proteins, prolinerich proteins, LRR domain containing protein, and GILT, were differentially expressed by $M$. saltuarius larvae, coupled with the difference in host preference of adults acting as vector of PWN, suggesting that larvae of $M$. saltuarius might confront with different bacterial challenges in their host environments.

Gene expression patterns identified in this study, might not just be altered by differential host plant diets, but also genetic variations in the sample populations [78], and local environments (i.e., temperature) [79]. In the present study, the two sample sites were approximately $40 \mathrm{~km}$ apart; thus, they may share similarities in their natural environment. Molecular identification of cytochrome c oxidase subunit I (COI) sequence distances of sampled individuals from the two sites were between 0.0 and 0.00897 , indicating little genetic divergence between $M$. saltuarius populations. Previously, similar results have been reported from comparable sampling strategies [26, 30, 80]. Therefore, the different gene expression patterns detected in the present study may be mainly caused by host plant diet. Further research is needed to verify that the DEGs detected in our analysis are due to host adaptation mechanisms in $M$. saltuarius.

\section{Conclusions}

In this study, firstly, we investigated the free amino and fatty acid composition and content of the host plants of
M. saltuarius larvae, i.e., P. koraiensis and P. tabuliformis. Compared with $P$. koraiensis, $P$. tabuliformis had a substantially higher content of various free amino acids, while the opposite trend was detected for fatty acid content. Then, we compared the transcriptional profiles of larval populations feeding on P. koraiensis and P. tabuliformis using PacBio Sequel II sequencing combined with Illumina sequencing. The results showed that genes relating to digestion, fatty acid synthesis, detoxification, oxidation-reduction, and stress response, as well as nutrients and energy sensing ability, were differentially expressed, possibly reflecting adaptive changes of $M$. saltuarius in response to different host diets. Additionally, genes coding for cuticle structure were differentially expressed, indicating that cuticle may be a target for plant defense. Differential regulation of genes associated with the antibacterial and immune response were also observed, suggesting that larvae of $M$. saltuarius may have evolved adaptations to cope with bacterial challenges in their host environments. The results from this study help to elucidate the underlying relationship between transcriptional plasticity and adaption mechanisms of herbivorous insects to host plants.

\section{Methods}

\section{Larvae and host plant sample collection}

Both male and female adults of $M$. saltuarius can spread exceeding $5 \mathrm{~km}$ throughout their whole life cycle [81]. To obtain representative $M$. saltuarius samples exclusively feeding on $P$. koraiensis, fourth-instar larvae of $M$. saltuarius were collected from Cangshi Forest Farm, located in Qingyuan Manchu Autonomous County, Liaoning Province $\left(41^{\circ} 59^{\prime} 24.72^{\prime \prime} \mathrm{N}, 124^{\circ} 31^{\prime} 41.17^{\prime \prime} \mathrm{E}\right)$, in September 2019. To obtain representative $M$. saltuarius samples exclusively feeding on $P$. tabuliformis, fourthinstar larvae of $M$. saltuarius were collected from Cangshi Village, Fushun County, Liaoning Province (41 $53^{\prime}$ $42.67^{\prime \prime} \mathrm{N}, 124^{\circ} 20^{\prime} 25.06^{\prime}$ 'E), on the same day in September, 2019. The two sample sites were approximately 40 $\mathrm{km}$ apart. Some of the larval samples were flash-frozen upon collection using liquid nitrogen, then transferred and stored at $-80^{\circ} \mathrm{C}$ for subsequent RNA extraction. The remaining samples were transferred alive to the laboratory and reared on their corresponding hosts until development into instars. In addition, three sample logs of each host plant were collected for amino and fatty acid composition and content analysis.

\section{Host plant amino and fatty acid composition and content analysis}

Three host plant samples from each Pinus spp. were used to investigate the composition and content of free amino acids according to the method described by Zeng et al. [82]. A $0.5 \mathrm{~g}$ sample powder was mixed with $50 \mathrm{~mL}$ 
hydrochloric acid $(0.005 \mathrm{~mol} / \mathrm{L})$ for ultrasonic extraction for $35 \mathrm{~min}$ at $40^{\circ} \mathrm{C}$ using ultrasonic cleaner. The homogenate was centrifuged at $15000 \mathrm{rpm}$ for $15 \mathrm{~min}$, then filtered with a $0.22 \mu \mathrm{m}$ membrane. Amino acid content analysis was carried out by an A300 amino acid analyzer (membraPure Bodenheim, Germany) with a column packed with ion-exchange resin. Amino acid concentration was calculated by calibrating with external standards (Sinopharm Chemical Reagent, Beijing, China).

Similarly, three host plant samples from each Pinus spp. were used to investigate the composition and content of fatty acids. The crude fat were extracted from the samples with petroleum ether (boiling point $40-60^{\circ} \mathrm{C}$ ) using a Soxhlet extractor. Fatty acid methyl ester (FAME) was prepared according to the PORIM Test Method [83]. The GC-MS systems (Trace1310/ISQ, Thermo Fisher Scientific, USA) equipped with a flame ionization detector and a TG-5 MS capillary column (30 $\mathrm{m} \times 0.25 \mathrm{~mm} \times 0.25 \mu \mathrm{m}$ ) were used for fatty acid composition analysis. Chromatographic parameters were set as follows: the initial temperature was $80^{\circ} \mathrm{C}$ for $1 \mathrm{~min}$ then raised to $200^{\circ} \mathrm{C}$ at a rate of $10^{\circ} \mathrm{C} / \mathrm{min}$, which was then increased to $250^{\circ} \mathrm{C}$ at a rate of $5^{\circ} \mathrm{C} / \mathrm{min}$, before being set to $270^{\circ} \mathrm{C}$ at a rate of $2{ }^{\circ} \mathrm{C} / \mathrm{min}$ and held for 3 min. The flow rate of carrier helium gas was $1.2 \mathrm{~mL} /$ min. FAMEs were identified by comparing their retention times with those of authentic standards (Sigma-Aldrich Chemie GmbH, Deisenhofen, Germany). Quantification of fatty acids was carried out based on the molecular weight of their corresponding FAMEs.

\section{Molecular identification of larvae}

DNA barcode of mitochondrial $\mathrm{COI}$ was employed to ensure that the collected larvae belonged to the same species of $M$. saltuarius. A total of six COI sequences (677 bp) was amplified using the primer set LCO149/ HCO2198 [84] from six representatives were obtained, i.e., three larvae feeding on each Pinus spp. The COI sequences of all individuals had little divergence, with the distances from 0.0 to 0.00897 falling within the range of genetic distance among $M$. saltuarius populations [85]. This, coupled with morphological characteristics, indicates that the larvae collected from different host plants all belong to $M$. saltuarius.

\section{Total RNA extraction and library construction}

Total RNA was extracted with TRIzol reagent (Life Technologies, USA) according to the manufacturer's instructions. RNA quality was assessed by a $1 \%$ agarose gel and the concentration was determined using a NanoDrop 2000 spectrophotometer (Thermo Fisher Scientific, USA). RNA integrity was examined using the Agilent Technologies 2100 Bioanalyzer system (Santa Clara, CA) with a RNA integrity number cutoff greater than 7 .
To obtain the complete information of all transcripts, SMRT-seq (PacBio) was applied in this study. The best RNA sample ( 2 populations $\times 3$ replicated samples) was selected and then pooled together in equal quantity for SMRT-seq. Full-length cDNA was synthesized using the SMRTer PCR cDNA Synthesis Kit (Biomarker, Beijing). PacBio Sequel II sequencing reactions from one SMRT cell $(1-6 \mathrm{~kb})$ were performed.

For Illumina RNA-seq, six RNA samples (2 populations $\times 3$ replicated samples) were used. Then, the six libraries for sequencing were generated using NEBNext ${ }^{\circ}$ Ultra $^{\text {mix }}$ RNA Library Prep Kit (NEB, Beverly, MA, USA) according to the manufacturer's recommendations. Subsequently, the prepared libraries were sequenced on an Illumina NovaSeq 6000 platform $(2 \times 150 \mathrm{bp})$.

\section{PacBio sequencing data processing}

SMRTlink 6.0 software was used to process the PacBio SMRT-seq raw reads. The CCS was obtained from the subreads.bam file. Sequencing adapters were trimmed, then clean CCS were classified into either full- or nonfull-length isoforms based on cDNA primers and poly-A tail signal. To improve consensus accuracy, Iterative Clustering for Error Correction (ICE) and Arrow algorithm (https://downloads.pacbcloud.com/public/ software/installers/smrtlink_5.0.1.9585.zip) were used to obtain high quality isoforms and full-length sequences. Additional nucleotide errors in consensus reads were corrected using the Illumina RNA-seq data with the software LoRDEC (Helsinki, Finland) [86]. BUSCO [87] was used to explore completeness according to conserved ortholog content.

\section{Structure analysis and annotation}

Transcripts with lengths more than 200 nucleotides and with more than two exons were selected as lncRNA candidates. A combination of four pervasive coding potential assessment approaches was built, including CPC [88], CNCI [89], CPAT [90], and Pfam database [91], to identify lncRNA. The transcription factors were predicted using AnimalTFDB 2.0 [92]. Simple sequence repeats (SSRs) of the transcriptome were identified using MIcroSAtellite identification tool v1.0 (MISA) (http:// pgrc.ipk-gatersleben.de/misa/).

Functional annotation of non-redundant transcripts was determined by searching in the public databases using BLASTX (v2.2.26) (cutoff E-value $\leq 1 \mathrm{e}-5$ ) [93], including $\mathrm{Nr}$, Nt (NCBI nucleotide sequences), KOG (euKaryotic Ortholog Groups), KEGG, Pfam, Swiss-Prot, and eggNOG (Non-supervised Orthologous Groups). Functional classification by GO analysis was conducted with the program Blast2GO (v2.5). 


\section{Mapping and differential gene expression analysis}

The pair-end Illumina reads were aligned to the reference transcriptome using Bowtie 2 (version 2.2.9) [94]. FPKM (fragments per kilobase of transcript per million fragments mapped) was used to estimate transcript expression levels in all samples [95]. Differential expression analysis between the larvae feeding on P. koraiensis and $P$. tabuliformis was implemented using the DEGseq $\mathrm{R}$ package (version 1.10.1) [96]. The threshold of DEGs was set at q-value $<0.05$ and absolute fold change $>2$. Heatmaps in this study were generated with a free online platform, OmicShare tools (http://www.omicshare.com/ tools/) based on normalized FPKM data.

GO enrichment analysis of the DEGs was implemented using the GOseq $\mathrm{R}$ package (version 1.10.0) based on the Wallenius non-central hyper-geometric distribution to adjust gene length bias in DEGs [97]. The statistical enrichment of DEGs in KEGG pathways was tested using KOBAS (KEGG Orthology-Based Annotation System) (version v2.0.12) [98].

\section{Validation of DEGs with qRT-PCR}

The real-time quantitative PCR (qRT-PCR) assay was conducted to validate the results of our transcriptome sequencing analysis. Reverse transcription was performed using the PrimeScript first strand cDNA synthesis kit (Takara, China) according to the manufacturer's protocol. The gene-specific primers were designed using Primer5 (PREMIER Biosoft International, USA). The ribosomal protein $S 3$ gene was used for internal control. Quantitative reactions were performed on the Real-Time PCR Detection System (ABI 7500, Applied Biosystems, USA) with the SYBR Premix Ex Taq ${ }^{\text {Tx }}$ Kit (Takara, China). The qPCR setting was as follows: $95^{\circ} \mathrm{C}$ for 5 min, followed by 40 cycles of $95^{\circ} \mathrm{C}$ for $10 \mathrm{~s}$ and $60^{\circ} \mathrm{C}$ for $30 \mathrm{~s}$, melt curves stages at $95^{\circ} \mathrm{C}$ for $15 \mathrm{~s}, 60^{\circ} \mathrm{C}$ for $1 \mathrm{~min}$, and $95^{\circ} \mathrm{C}$ for $15 \mathrm{~s}$. To check reproducibility, the qPCR reaction for each sample was performed in triplicate. $\log _{2}($ fold change) $\mathrm{qPCR}$ was calculated by $-\Delta \Delta \mathrm{Ct}=-$ $\left[\left(\mathrm{Ct}_{\mathrm{Pt}}-\mathrm{Ct} \mathrm{t}_{\mathrm{nom}}\right)-\left(\mathrm{Ct}_{\mathrm{Pk}}-\mathrm{Ct}_{\mathrm{nom}}\right)\right][99]$.

\footnotetext{
Abbreviations

ABC transporters: ATP-binding cassette transporters; AKR: Aldo-keto reductases; $\mathrm{ALDH}$ : Aldehyde dehydrogenase; AMPK: AMP-activated protein kinase; BUSCO: Benchmarking universal single-copy orthologs; CAT: Catalase; CCS: Circular consensus sequence; cDNA: Complementary DNA; CE: Carboxylesterases; CNCl: Coding-Non-Coding Index; COI: Cytochrome C oxidase subunit I; CPAT: Coding Potential Assessment Tool; CPC: Coding Potential Calculator; DEG: Differentially expressed gene; DNA: Deoxyribonucleic acid; ELOVL: Elongation of very long chain fatty acids protein; FAME: Fatty acid methyl ester; FAR: Fatty acyl-CoA reductase; FAS: Fatty acid synthase; FLNC read: Full-length non-chimera read; FPKM: Fragments per kilobase of transcript per million fragments mapped; GC content: Guanine-cytosine content; GC-MS: Gas Chromatography-Mass Spectromete; GILT: Gamma-interferon-inducible lysosomal thiol reductase; GO: Gene Ontology; GOBP: General odorant-binding protein; GPx: Glutathione peroxidase; Hsp: Heat shock proteins; ICE: Iterative Clustering for Error Correction; KEGG: Kyoto encyclopedia of genes and
}

genomes; KOG: Eukaryotic orthologous groups; IncRNA: Long noncoding RNA; LPS: Lipopolysaccharide; LRR: Leucine-rich repeat; MISA: MIcroSAtellite identification tool; mRNA: Messenger RNA; MSRA: Peptide methionine sulfoxide reductase; Nr: NCBI non-redundant protein sequences; Nt: NCBI nucleotide sequences; OBP: Odorant-binding protein; P450: Cytochrome P450 monooxygenases; PCR: Polymerase chain reaction; Pfam: Protein family; PORIM: Palm Oil Research Institute of Malaysia; Prx: Peroxiredoxin; PWN: Pine wood nematode; RNA: Ribonucleic acid; RNA-Seq: RNA sequencing; ROS: Reactive oxygen species; qRT-PCR: Real-time quantitative PCR; SLC: Solute carriers; SMRT-seq: Single-molecule real-time sequencing; SNO: Senecionine N-oxygenase; SOD: Superoxide dismutase; SSR: Simple sequence repeats; STKs: Serine/threonine kinases; STPs: Serine/threonine phosphatases; TF: Transcription factor; TOR: Target of rapamycin; UGT: UDPglycosyltransferases; UV: Ultraviolet

\section{Supplementary Information}

The online version contains supplementary material available at https://doi. org/10.1186/s12864-021-07498-1.

\section{Additional file 1: Figure S1. Read length of CCS and FLNC. a CCS. b} FLNC.

Additional file 2: Figure S2. The completeness of transcripts assessed by benchmarking universal single-copy ortholog (BUSCO). The $\mathrm{x}$-axis represents the percentage of detected BUSCOs. The light blue diamond represents the complete $(\mathrm{C})$ and single-copy $(\mathrm{S})$ genes; the dark blue represents complete and duplicated (D) genes; the yellow diamond represents fragmented (F) genes; the red diamond represents the missing (M) genes. Total number of core genes queried was 1066.

Additional file 3: Table S1. Annotation of the 32,304 non-redundant transcripts.

Additional file 4: Figure S3. Species distribution of the top BLAST hits of the total homologous sequences.

Additional file 5: Figure S4. Gene ontology classification of nonredundant transcripts. The 13,144 transcripts were classified into three functional categories: molecular function, biological process and cellular component.

Additional file 6: Figure S5. KEGG pathway distributions of nonredundant transcripts. The genes according to KEGG metabolic pathway involved was divided into six branches: Metabolism, Genetic information processing, Environmental information processing, Cellular processes, Organismal systems, and Human disease.

Additional file 7: Figure S6. Number of transcript factors identified in the present study.

Additional file 8: Table S2. Summary of SSRs identified in the transcriptome of Monochamus saltuarius.

Additional file 9: Table S3. Annotation of the 2166 differentially expressed genes.

Additional file 10: Figure S7. Cluster analysis of differentially expressed genes. Different colors indicate different levels of gene expression. The firebrick color indicates upregulated expression, whereas the navy color indicates downregulated expression. Pk: the larvae feeding on Pinus koraiensis; Pt: the larvae feeding on P. tabuliformis.

Additional file 11: Table S4. List of KEGG pathways with $P$-value $<0.05$ Additional file 12: Figure S8. GO enrichment of differentially expressed genes. The 20 most enriched GO terms are shown together with their $-\log _{10}(\mathrm{Q}$-value) and number of genes.

Additional file 13: Table S5. Primers used in GRT-PCR.

\section{Acknowledgments}

We would like to express our deep gratitude to Haiying Yu (General Station of Forest and Grassland Pest Management, National Forestry and Grassland Administration, China), Dr. Xueqing Yang (Shenyang Agricultural University, China) and three anonymous workers at Cangshi Forest Farm, located in Qingyuan Manchu Autonomous County, Liaoning Province for their help with specimen collection. 


\section{Authors' contributions}

$\mathrm{ZH}$ and $\mathrm{SZ}$ designed the study. ZH and FS performed the experiments and analyzed the data. SG and HW provided help with the experiments. ZH wrote the manuscript. SZ, JT and LR discussed the results and edited the manuscript. All authors read and approved the final manuscript.

\section{Funding}

This research was funded by the Fundamental Research Funds for the Central Universities (Grant No. BLX201902) (for Z.H.), the National Key Research and Development Program of China (2018YFD0600200) (for S.Z.), and the Major Project of Liaoning Province Programs for Fundamental Research and Development (2019JH2/1020001) (for H.W.). The funders had no role in the design of the study and collection, analysis, and interpretation of data and in writing the manuscript.

\section{Availability of data and materials}

Raw PacBio SMRT sequences and Illumina RNA-Seq data for this study have been deposited in the National Center for Biotechnology Information (NCBI) Sequence Read Archive (SRA) (http://www.ncbi.nlm.nih.gov/sra) (BioProject: PRJNA666339). All COI sequences obtained were submitted to NCBI GenBank (https://www.ncbi.nlm.nih.gov/genbank) (accession numbers: MW074321MW074326)

\section{Declarations}

\section{Ethics approval and consent to participate}

No specific permits were required for insect collection in the selected locations. The sampling locations are not privately owned or natural protected areas. The study species is not included in the "List of Protected Animals in China", and their collection is legal in China.

\section{Consent for publication}

Not applicable.

\section{Competing interests}

The authors declare that they have no competing interests.

\section{Author details}

${ }^{1}$ Key Laboratory of Beijing for the Control of Forest Pests, Beijing Forestry University, Beijing, China. ${ }^{2}$ Liaoning Provincial Key Laboratory of Dangerous Forest Pest Management and Control, Shenyang, China.

\section{Received: 15 November 2020 Accepted: 2 March 2021} Published online: 16 March 2021

\section{References}

1. Janz N, Nylin S, Wahlberg N. Diversity begets diversity: host expansions and the diversification of plant-feeding insects. BMC Evol Biol. 2006;6:4

2. de Panis DN, Padró J, Furió-Tarí P, Tarazona S, Carmona PSM, Soto IM, et al. Transcriptome modulation during host shift is driven by secondary metabolites in desert Drosophila. Mol Ecol. 2016;25:4534-50.

3. Vogel H, Musser RO, Celorio-Mancera M. Transcriptome responses in herbivorous insects towards host plant and toxin feeding. In: Voelckel C, Jander G, editors. Annual plant review vol. 47 insect-plant interaction. Chichester: Wiley; 2014. p. 197-233.

4. Dermauw W, Wybouw N, Rombauts S, Menten B, Vontas J, Grbić M, et al. A link between host plant adaptation and pesticide resistance in the polyphagous spider mite Tetranychus urticae. Proc Natl Acad Sci U S A. 2013;110:E113-22.

5. Zhao BG, Futai K, Sutherland JR, Takeuchi Y. Pine wilt disease. Tokyo: Springer; 2008.

6. Kishi Y. Pine wood nematode and the Japanese pine sawyer. Tokyo: Thomas Company Limited; 1995.

7. Kwon TS, Lim JH, Sim SJ, Kwon YD, Son SK, Lee KY, et al. Distribution patterns of Monochamus alternatus and M. saltuarius (Coleoptera: Cerambycidae) in Korea. J Kor For Soc. 2006;95:543-50.

8. Heisuke S, Takeshi S, Mitsunori K. Transmission of Bursaphelenchus xylophilus (Steiner et Buhrer) Nickle (Nematoda: Aphelenchoididae) by Monochamus saltuarius (Gebler) (Coleoptera, Cerambycidae). J Jpn For Soc. 1987;69:492-6.
9. KFRI [Korea Forest Research Institute]. Annual report of monitoring for forest insect pest and diseases in Korea. Seoul: Korea Forest Research Institute; 2007

10. $\mathrm{Yu} \mathrm{H}, \mathrm{Wu} \mathrm{H}$. New host plants and new vector insects found in pine wood nematode in Liaoning. For Pest Dis. 2018;37:61.

11. Pan L, Li Y, Cui R, Liu Z, Zhang X. Monochamus saltuarius endangers Pinus tabuliformis Carr. and carries Bursaphelenchus xylophilus (Steiner and Buhrer) in China. Forests. 2020;11:1051.

12. Li M, Li H, Sheng RC, Sun H, Sun SH, Chen FM. The first record of Monochamus saltuarius (Coleoptera; Cerambycidae) as vector of Bursaphelenchus xylophilus and its new potential hosts in China. Insects. 2020;11:636.

13. Kang K-S, Lindgren D. Fertility variation among clones of Korean pine (Pinus koraiensis $\mathrm{S}$. et Z.) and its implications on seed orchard management. For Genet. 1999;6:191-200.

14. Shin SC. Pine wilt disease in Korea. In: Zhao BG, Futai K, Sutherland JR, Takeuchi Y, editors. Pine wilt disease. Tokyo: Springer; 2008. p. 26-32.

15. Han J-H, Kim HK, Kang WJ, Kim G-H. Feeding and oviposition preference of the Sakhalin pine sawyer Monochamus saltuarius (Coleoptera: Cerambycidae) for various tree species. Entomol Res. 2016;46:331-6.

16. Pan J, Li J, Dong Y, Lou J, Zhang T, Yu H. Feeding preference of the Monochamus saltuarius Gebler (Coleoptera: Cerambycidae) for Pinus koraiensis, P. tabulaeformis and Larix kaempferi. For Pest Dis. 2020;39:19-22.

17. Ikeda T, Enda N, Yamane A, Oda K, Toyoda T. Attractants for the Japanese pine sawyer, Monochamus alternatus Hope (Coleoptera: Cerambycidae). Appl Entomol Zool. 1980;15:358-61.

18. Lee SM, Hong DK, Jang SH, Lee KY, Lee CW. Synergistic attraction of pine sawyer Monochamus saltuarius (Coleoptera: Cerambycidae) to monochamol and a-pinene. Entomol Res. 2016;47:125-8.

19. Kang SH, Kim MK, Yang JO, Yoon CM, Goh SH, Shin SC, et al. Attraction effects to various conditions of Pinus koraiensis against pine sawyer, Monochamus saltuarius (Coleoptera: Cerambycidae). J Korean Soc Appl Biol Chem. 2009:52:456-65.

20. Yoon C, Shin YH, Yang JO, Han JH, Kim GH. Pinus koraiensis twigs affect Monochamus saltuarius (Coleoptera: Cerambycidae) longevity and reproduction. J Asia Pac Entomol. 2011;14:327-33.

21. Ginzel MD, Hanks LM. Role of host plant volatiles in mate location for three species of longhorned beetles. J Chem Ecol. 2005;31:213-7.

22. Rudolf VHW, Rödel MO. Oviposition site selection in a complex and variable environment: the role of habitat quality and conspecific cues. Oecologia. 2005;142:316-25.

23. Refsnider JM, Janzen FJ. Putting eggs in one basket: ecological and evolutionary hypotheses for variation in oviposition site choice. Annu Rev Ecol Syst. 2010;41:39-57.

24. Morris DW. Toward an ecological synthesis: a case for habitat selection. Oecologia. 2003;136:1-13.

25. Hoang K, Matzkin LM, Bono JM. Transcriptional variation associated with cactus host plant adaptation in Drosophila mettleri populations. Mol Ecol. 2015;24:5186-99.

26. Zhong H, Li F, Chen J, Zhang J, Li F. Comparative transcriptome analysis reveals host-associated differentiation in Chilo suppressalis (Lepidoptera: Crambidae). Sci Rep. 2017;7:13778.

27. Matzkin LM. Population transcriptomics of cactus host shifts in Drosophila mojavensis. Mol Ecol. 2012;21:2428-39.

28. Mason CJ, Scully ED, Geib SM, Hoover K. Contrasting diets reveal metabolic plasticity in the tree-killing beetle, Anoplophora glabripennis (Cerambycidae: Lamiinae). Sci Rep. 2016;6:33813.

29. Scully ED, Geib SM, Mason CJ, Carlson JE, Ming T, Chen HY, et al. Host-plant induced changes in microbial community structure and midgut gene expression in an invasive polyphage (Anoplophora glabripennis). Sci Rep. 2018:8:9620.

30. Hou Z, Wei C. De novo comparative transcriptome analysis of a rare cicada, with identification of candidate genes related to adaptation to a novel host plant and drier habitats. BMC Genomics. 2019;20:182

31. Boerjan B, Cardoen D, Verdonck R, Caers J, Schoofs L. Insect omics research coming of age1. Can J Zool. 2012;90:440-55.

32. Oates C, Denby K, Myburg A, Slippers B, Naidoo S. Insect gallers and their plant hosts: from omics data to systems biology. Int J Mol Sci. 2016;17:1891.

33. Treutlein B, Gokce O, Quake SR, Südhof TC. Cartography of neurexin alternative splicing mapped by single-molecule long-read mRNA sequencing. Proc Natl Acad Sci U S A. 2014;111:E1291-9. 
34. Hackl T, Hedrich R, Schultz J, Forster F. Proovread: large-scale high-accuracy PacBio correction through iterative short read consensus. Bioinformatics. 2014;30:3004-11.

35. Sharon D, Tilgner $H$, Grubert F, Snyder M. A single-molecule long-read survey of the human transcriptome. Nat Biotechnol. 2013;31:1009-14.

36. Zhang J, Guan W, Huang C, Hu Y, Chen Y, Guo J, et al. Combining nextgeneration sequencing and single-molecule sequencing to explore brown plant hopper responses to contrasting genotypes of japonica rice. BMC Genomics. 2019;20:682

37. Hultmark D. Immune reactions in Drosophila and other insects: a model for innate immunity. Trends Genet. 1993;9:178-83.

38. Sagisaka A, Miyanoshita A, Ishibashi J, Yamakawa M. Purification, characterization and gene expression of a glycine and proline-rich antibacterial protein family from larvae of a beetle, Allomyrina dichotoma. Insect Mol Biol. 2001;10:293-302.

39. Herde M, Howe GA. Host plant-specific remodeling of midgut physiology in the generalist insect herbivore Trichoplusia ni. Insect Biochem Mol Biol. 2014;50:58-67.

40. Roy A, Walker WB, Vogel H, Chattington S, Larsson MC, Anderson P, et al. Diet dependent metabolic responses in three generalist insect herbivores Spodoptera spp. Insect Biochem Mol Biol. 2016:71:91-105.

41. Ragland GJ, Almskaar K, Vertacnik KL, Gough HM, Feder JL, Hahn DA, et al. Differences in performance and transcriptome-wide gene expression associated with Rhagoletis (Diptera: Tephritidae) larvae feeding in alternate host fruit environments. Mol Ecol. 2015;24:2759-76.

42. Chikate YR, Tamhane VA, Joshi RS, Gupta VS, Giri AP. Differential protease activity augments polyphagy in Helicoverpa armigera. Insect Mol Biol. 2013;22:258-72.

43. Celorio-Mancera MP, Wheat CW, Vogel H, Söderlind L, Janz N, Nylin S. Mechanisms of macroevolution: polyphagous plasticity in butterfly larvae revealed by RNA-Seq. Mol Ecol. 2013;22:4884-95.

44. Loewith $\mathrm{R}$, Hall MN. Target of rapamycin (TOR) in nutrient signaling and growth control. Genetics. 2011;189:1177-201.

45. Ali Y, Ruan K, Zhai RG. Drosophila models of tauopathy. In: LeDoux MS, editor. Movement disorders: genetics and models. 2nd ed: Academic Press; 2015. p. 829-48

46. Francez-Charlot A, Kaczmarczyk A, Fischer H-M, Vorholt JA. The general stress response in alphaproteobacteria. Trends Microbiol. 2015;23:164-71.

47. Mijakovic I, Grangeasse C, Turgay K. Exploring the diversity of protein modifications: special bacterial phosphorylation systems. FEMS Microbiol Rev. 2016:40:398-417.

48. Janczarek M, Vinardell J-M, Lipa P, Karaś M. Hanks-type serine/threonine protein kinases and phosphatases in bacteria: roles in signaling and adaptation to various environments. Int J Mol Sci. 2018;19:2872.

49. Hardie DG. AMP-activated protein kinase: an energy sensor that regulates all aspects of cell function. Genes Dev. 2011;25:1895-908.

50. González A, Hall MN, Lin S-C, Hardie DG. AMPK and TOR: the yin and Yang of cellular nutrient sensing and growth control. Cell Metab. 2020;31:472-92.

51. Stanley-Samuelson DW, Jurenka RA, Cripps C, Blomquist GJ, de Renobales M. Fatty acids in insects: composition, metabolism, and biological significance. Arch Insect Biochem Physiol. 1988;9:1-33.

52. Chung H, Loehlin DW, Dufour HD, Vaccarro K, Millar JG, Carroll SB. A single gene affects both ecological divergence and mate choice in Drosophila. Science. 2014:343:1148-51.

53. Pei X-J, Chen N, Bai Y, Qiao J-W, Li S, Fan Y-L, et al. BgFas1: a fatty acid synthase gene required for both hydrocarbon and cuticular fatty acid biosynthesis in the German cockroach, Blattella germanica (L.). Insect Biochem Mol Biol. 2019:112:103203.

54. Li L, Jiang Y, Liu Z, You L, Wu Y, Xu B, et al. Jinggangmycin increases fecundity of the brown planthopper, Nilaparvata lugens (Stål) via fatty acid synthase gene expression. J Proteome. 2016;130:140-9.

55. Sassa T, Kihara A. Metabolism of very long-chain fatty acids: genes and pathophysiology. Biomol Ther. 2014;22:83-92.

56. Leonard A, Pereira SL, Sprecher H, Huang Y-S. Elongation of long-chain fatty acids. Prog Lipid Res. 2004:43:36-54

57. Moto K, Yoshiga T, Yamamoto M, Takahashi S, Okano K, Ando T, et al. Pheromone gland-specific fatty-acyl reductase of the silkmoth, Bombyx mori. Proc Natl Acad Sci U S A. 2003;100:9156-61.

58. Hu $Y-H$, Chen $X-M$, Yang $P$, Ding $W-F$. Characterization and functional assay of a fatty acyl-CoA reductase gene in the scale insect, Ericerus pela Chavannes (Hemiptera: Coccoidae). Arch Insect Biochem Physiol. 2018;97: e21445.
59. Li DT, Dai YT, Chen X, Wang XQ, Li ZD, Moussian B, et al. Ten fatty acyl-CoA reductase family genes were essential for the survival of the destructive rice pest, Nilaparvata lugens. Pest Manag Sci. 2020;76:2304-15.

60. Xu LT, Lu M, Sun JH. Invasive bark beetle-associated microbes degrade a host defensive monoterpene. Insect Sci. 2016;23:183-90.

61. Gao Y, Jin YJ, Li HD, Chen HJ. Volatile organic compounds and their roles in bacteriostasis in five conifer species. J Integr Plant Biol. 2005;47:499-507.

62. Xu Q, Sun XT, Lu PF, Luo YQ, Shi J. Volatile profiles of three tree species in the northeastern China and associated effects on Sirex noctilio activity. J Plant Interact. 2019;14:334-9.

63. Grbić M, Leeuwen TV, Clark RM, Rombauts S, Rouzé P, Grbić V, et al. The genome of Tetranychus urticae reveals herbivorous pest adaptations. Nature. 2011:479:487-92.

64. Sun H, Pu J, Chen F, Wang J, Han Z. Multiple ATP-binding cassette transporters are involved in insecticide resistance in the small brown planthopper, Laodelphax striatellus. Insect Mol Biol. 2017;26:343-55.

65. Danquah KO, Gyamfi D. Alcohol and aldehyde dehydrogenases: molecular aspects. In: Patel VB, editor. Molecular aspects of alcohol and nutrition: Academic Press; 2016. p. 25-43.

66. Naumann C, Hartmann T, Ober D. Evolutionary recruitment of a flavindependent monooxygenase for the detoxification of host plant-acquired pyrrolizidine alkaloids in the alkaloid-defended arctiid moth Tyria jacobaeae. Proc Natl Acad Sci U S A. 2002;99:6085-90.

67. Penning TM. The aldo-keto reductases (AKRs): overview. Chem Biol Interact. 2015;234:236-46.

68. Mittapalli O, Neal JJ, Shukle RH. Antioxidant defense response in a galling insect. Proc Natl Acad Sci U S A. 2007;104:1889-94.

69. Barbehenn RV. Gut-based antioxidant enzymes in a polyphagous and a graminivorous grasshopper. J Chem Ecol. 2002;28:1329-47.

70. Hillyer JF. Insect immunology and hematopoiesis. Dev Comp Immunol. 2016;58:102-18

71. Dunn PE. Biochemical aspects of insect immunology. Annu Rev Entomol. 1986;31:321-39.

72. Hoffmann JA. Innate immunity of insects. Curr Opin Immunol. 1995;7:4-10.

73. Zhang H, Li S, Wang F, Xiang J, Li F. Identification and functional study of an LRR domain containing membrane protein in Litopenaeus vannamei. Dev Comp Immunol. 2020;109:103713.

74. Zhu Y, Ragan EJ, Kanost MR. Leureptin: a soluble, extracellular leucine-rich repeat protein from Manduca sexta that binds lipopolysaccharide. Insect Biochem Mol Biol. 2010:40:713-22.

75. Rausch MP, Hastings KT. Diverse cellular and organismal functions of the Iysosomal thiol reductase GILT. Mol Immunol. 2015;68:124-8.

76. Proença DN, Francisco R, Kublik S, Schöler A, Vestergaard G, Schloter M, et al. The microbiome of endophytic, wood colonizing bacteria from pine trees as affected by pine wilt disease. Sci Rep. 2017;7:4205.

77. Guo Y, Lin Q, Chen L, Carballar-Lejarazú R, Zhang A, Shao E, et al. Characterization of bacterial communities associated with the pinewood nematode insect vector Monochamus alternatus Hope and the host tree Pinus massoniana. BMC Genomics. 2020:21:337.

78. Giorello FM, Feijoo M, D'Elía G, Naya DE, Valdez L, Opazo JC, et al. An association between differential expression and genetic divergence in the Patagonian olive mouse (Abrothrix olivacea). Mol Ecol. 2018;27:3274-86.

79. Huang HJ, Xue J, Zhuo JC, Cheng RL, Xu HJ, Zhang CX. Comparative analysis of the transcriptional responses to low and high temperatures in three rice planthopper species. Mol Ecol. 2017;26:2726-37.

80. Liu Y, Qi M, Dietrich CH, He Z, Wei C. Comparative sialotranscriptome analysis of the rare Chinese cicada Subpsaltria yangi, with identification of candidate genes related to host-plant adaptation. Int J Biol Macromol. 2019;130:323-32

81. Kwon HJ, Jung J-K, Jung $\mathrm{C}$, Han H, Koh S-H. Dispersal capacity of Monochamus saltuarius on flight mills. Entomol Exp Appl. 2018;166:420-7.

82. Zeng Y, Cai W, Shao X. Quantitative analysis of 17 amino acids in tobacco leaves using an amino acid analyzer and chemometric resolution. J Sep Sci. 2015;38:2053-8.

83. PORIM. Official test method. Bandar Baru Bangi: Palm Oil Research of Malaysia; 1995.

84. Folmer O, Black M, Hoeh W, Lutz R, Vrijenhoek R. DNA primers for amplification of mitochondrial cytochrome c oxidase subunit I from diverse metazoan invertebrates. Mol Mar Biol Biotechnol. 1994:3:294-9.

85. Jeon J, Byun B, Cho Y, Woo S, Kim C, Lim J, et al. Intraspecific diversity of Monochamus saltuarius (Gebler) based on DNA barcode analysis. J Asia Pac Biodivers. 2015;8:305-8. 
86. Salmela L, Rivals E. LoRDEC: accurate and efficient long read error correction. Bioinformatics. 2014;30:3506-14.

87. Simão FA, Waterhouse RM, Panagiotis I, Kriventseva EV, Zdobnov EM. BUSCO: assessing genome assembly and annotation completeness with single-copy orthologs. Bioinformatics. 2015;31:3210-2.

88. Kong L, Zhang Y, Ye Z-Q, Liu X-Q, Zhao S-Q, Wei L, et al. CPC: assess the protein-coding potential of transcripts using sequence features and support vector machine. Nucleic Acids Res. 2007;35:W345-9.

89. Sun L, Luo H, Bu D, Zhao G, Yu K, Zhang C, et al. Utilizing sequence intrinsic composition to classify protein-coding and long non-coding transcripts. Nucleic Acids Res. 2013:41:e166.

90. Wang L, Park HJ, Dasari S, Wang S, Kocher J-P, Li W. CPAT: coding-potential assessment tool using an alignment-free logistic regression model. Nucleic Acids Res. 2013;41:e74.

91. Finn RD, Coggill P, Eberhardt RY, Eddy SR, Mistry J, Mitchell AL, et al. The Pfam protein families database: towards a more sustainable future. Nucleic Acids Res. 2016;44:D279-85.

92. Zhang H-M, Liu T, Liu C-J, Song S, Zhang X, Liu W, et al. AnimalTFDB 2.0: a resource for expression, prediction and functional study of animal transcription factors. Nucleic Acids Res. 2015;43:D76-81.

93. Altschul SF, Madden TL, Schäffer AA, Zhang J, Zhang Z, Miller W, et al. Gapped BLAST and PSI-BLAST: a new generation of protein database search programs. Nucleic Acids Res. 1997;25:3389-402.

94. Langmead B, Salzberg SL. Fast gapped-read alignment with bowtie 2. Nat Methods. 2012;9:357-9.

95. Trapnell C, Williams BA, Pertea G, Mortazavi A, Kwan G, van Baren MJ, et al. Transcript assembly and quantification by RNA-Seq reveals unannotated transcripts and isoform switching during cell differentiation. Nat Biotechnol. 2010;28:511-5

96. Wang L, Feng Z, Wang $X$, Wang $X$, Zhang $X$. DEGseq: an $R$ package for identifying differentially expressed genes from RNA-seq data. Bioinformatics. 2010;26:136-8

97. Young MD, Wakefield MJ, Smyth GK, Oshlack A. Gene ontology analysis for RNA-seq: accounting for selection bias. Genome Biol. 2010;11:R14.

98. Mao X, Cai T, Olyarchuk JG, Wei L. Automated genome annotation and pathway identification using the KEGG Orthology (KO) as a controlled vocabulary. Bioinformatics. 2005:21:3787-93.

99. Song G-Q, Chen Q. Comparative transcriptome analysis of nonchilled, chilled, and late-pink bud reveals flowering pathway genes involved in chilling-mediated flowering in blueberry. BMC Plant Biol. 2018;18:98.

\section{Publisher's Note}

Springer Nature remains neutral with regard to jurisdictional claims in published maps and institutional affiliations.

Ready to submit your research? Choose BMC and benefit from:

- fast, convenient online submission

- thorough peer review by experienced researchers in your field

- rapid publication on acceptance

- support for research data, including large and complex data types

- gold Open Access which fosters wider collaboration and increased citations

- maximum visibility for your research: over $100 \mathrm{M}$ website views per year

At $\mathrm{BMC}$, research is always in progress.

Learn more biomedcentral.com/submissions 